\title{
Ceratocystis fimbriata agente causal de la enfermedad de marchitez vascular de Tectona grandis L.f. (teca) en Ecuador
}

\author{
(Ceratocystis fimbriata causative agent of vascular wilt disease of Tectona grandis L.f. \\ (teak) in Ecuador)
}

\begin{abstract}
Belezaca-Pinargote, C. ${ }^{1,3 *}$, Solano-Apuntes, E. ${ }^{1}$, López-Tobar, R.1, Cóndor-Jiménez, M. ${ }^{2}$, Beltrán-Castro, $\mathrm{F}^{3}$, Díaz-Navarrete, $\mathrm{P}^{4}$

${ }^{1}$ Universidad Técnica Estatal de Quevedo, Carrera de Ingeniería Forestal, Quevedo, Ecuador.

${ }^{2}$ ENDESA - BOTROSA, Ecuador.

${ }^{3}$ Universidad Técnica de Babahoyo, Carrera de Ingeniería Agropecuaria, Babahoyo, Ecuador.

${ }^{4}$ Universidad Católica de Temuco, Facultad de Ingeniería, Escuela de Procesos Industriales, Laboratorio de Bioprocesos, Temuco, Chile. *Autor para correspondencia: cbelezaca@uteq.edu.ec
\end{abstract}

RECIBIDO: 25 de febrero 2020 APROBADO: 23 de junio 2020

DOI: 10.22370/bolmicol.2020.35.1.2401

Palabras claves: Fusarium, patogénesis, postulados de Koch, volumen aparente de necrosis. Keywords: Fusarium, pathogenesis, Koch postulates, apparent volume of necrosis.

\section{RESUMEN}

Tectona grandis L.f. es una especie forestal de madera dura que, a pesar de no ser nativa de América, su plasticidad de adaptación ha permitido que en Ecuador se establezcan plantaciones intensivas con fines de exportación. Una compleja enfermedad con características de marchitez vascular está matando miles de árboles de diferentes edades en el país. Se planteó conocer el agente fitopatógeno causante de la patogénesis y muerte de los árboles. Se aislaron los hongos Fusarium sp. y Ceratocystis fimbriata Ellis \& Halst. desde árboles enfermos. A nivel de invernadero se plantearon los postulados de Koch, para el efecto 30 plantas de T. grandis de cuatro meses de edad por cada tratamiento, se inocularon con los microrganismos como se describe a continuación: $\mathrm{T} 1=C$. fimbriata, $\mathrm{T} 2=$ Fusarium sp., T3 = C. fimbriata + Fusarium sp., T4 = agaragar (control). Se empleó un diseño completo al azar (DCA) y las plantas se evaluaron a los 45 días después de inoculadas. Los tratamientos $C$. fimbriata, y C. fimbriata + Fusarium sp., mostraron mayores volúmenes aparentes de necrosis, con $1.52 \mathrm{~cm}^{3}$ y $1.93 \mathrm{~cm}^{3}$, y plantas muertas por la infección durante el tiempo de evaluación, mientras que Fusarium sp. mostró baja o nula patogenicidad y comportamiento similar al control, con $0.27 \mathrm{~cm}^{3}$ y $0.16 \mathrm{~cm}^{3}$ respectivamente. Estos resultados sugieren que el agente causal de la enfermedad de marchitez vascular en $T$. grandis es $C$. fimbriata y sería el primer reporte del fitopatógeno atacando teca en Ecuador. 
Ceratocystis fimbriata agente causal de la enfermedad de marchitez vascular de Tectona grandis L.f. (teca) en Ecuador Belezaca-Pinargote, $\mathrm{C}$.

\begin{abstract}
Tectona grandis L.f. it is a hardwood forest species, which despite not being native to America, its adaptive plasticity has allowed intensive plantations to be established for export purposes in Ecuador. A complex disease with characteristics of vascular wilt is killing thousands of trees of different ages in the country. It was proposed to know the phytopathogenic agent causing the pathogenesis and death of the trees. Fusarium sp. and Ceratocystis fimbriata Ellis \& Halst. were isolated from diseased trees. At the greenhouse level, Koch's postulates were proposed, for the effect 30 four month old $T$. grandis plants for each treatment were inoculated with the microorganisms as described below: $\mathrm{T} 1=C$. fimbriata, $\mathrm{T} 2=$ Fusarium $\mathrm{sp} ., \mathrm{T} 3=C$. fimbriata + Fusarium sp., T4 = agar-agar (control). A complete randomized design (DCA) was used and the plants were evaluated 45 days after inoculation. The treatments $C$. fimbriata, and $C$. fimbriata + Fusarium sp. showed higher apparent volumes of necrosis, with $1.52 \mathrm{~cm}^{3}$ and $1.93 \mathrm{~cm}^{3}$, and plants killed by the infection during the evaluation time, while Fusarium sp. showed low or no pathogenicity and behavior similar to the control, with $0.27 \mathrm{~cm}^{3}$ and 0.16 $\mathrm{cm}^{3}$ respectively. These results suggest that the causative agent of vascular wilt disease in $T$. grandis is $C$. fimbriata and it would be the first report of phytopathogen attacking teak in Ecuador.
\end{abstract}

\section{INTRODUCCIÓN}

La teca (Tectona grandis L.f.), es una especie forestal exótica, originaria del sureste asiático (india, Myanmar, Tailandia y Laos) ${ }^{\mathbf{1}}$ introducida a Ecuador en la década de 1950, convirtiéndose desde entonces en una especie de importancia económica para el país ${ }^{2}$. Se estima que actualmente existen 45000 ha de teca distribuidas en las regiones tropicales de Ecuador $^{\mathbf{3}}$, establecidas principalmente como plantaciones monoespecíficas (puras) con materiales genéticos mejorados (semillas) introducidos desde Costa Rica ${ }^{2}$.

Durante casi seis décadas se ha plantado esta especie forestal en Ecuador con extraordinarios resultados, no obstante, la masificación de plantaciones puras ha desencadenado en la presencia de problemas fitosanitarios serios que se han acentuado durante la última década. Actualmente una enfermedad con características de marchitez vascular está matando miles de árboles de teca en pie, causando un detrimento prematuro del vigor y estado sanitario de los árboles, generando pérdidas económicas significativas a silvicultores e inversionistas y poniendo en riesgo la viabilidad de la especie en el país ${ }^{2}$. En este sentido, Belezaca-Pinargote et al. (2018) ${ }^{2}$ estudiaron el problema y descubrieron que la enfermedad provoca clorosis, pérdida de turgencia en hojas, secamiento de yemas de crecimiento y ramas, notorio detrimento del vigor frente a árboles sanos debido a la perdida de área fotosintética que estimula la emisión de brotes epicormicos en el fuste, hasta que finalmente el árbol muere en pie. Análisis fitopatológicos permitieron aislar a Fusarium spp. y Ceratocystis fimbriata Ellis \& Halst., asociados a tejidos necrosados de árboles enfermos, sin que hasta el momento se conozca cuál de estos microorganismos ocasionan la patología o si actúan asociados ${ }^{2,4}$.

Considerando que en la patogénesis están asociados dos microorganismos altamente fitopatogénicos, se planteó identificar cuál de ellos es el agente causal o si actúan en sinergia para generar la enfermedad de la teca en Ecuador.

\section{MATERIALES Y MÉTODOS}

\section{Localización de sitio experimental.}

La investigación se llevó a cabo en el Invernadero y Laboratorio de Microbiología Ambiental y Vegetal de la Universidad Técnica Estatal de Quevedo (UTEQ), ubicado en el 
Ceratocystis fimbriata agente causal de la enfermedad de marchitez vascular de Tectona grandis L.f. (teca) en Ecuador Belezaca-Pinargote, $\mathrm{C}$.

campus central "Ing. Manuel Haz Álvarez", Km 1.5, vía Quevedo - Quito.

\section{Preparación del inóculo y pruebas de patogenicidad.}

Se empleó la colección de cepas de los hongos Ceratocystis fimbriata y Fusarium spp., colectadas desde árboles enfermos de teca por Ávila-Loor $(2016)^{4}$, que reposan en el laboratorio de Microbiología Ambiental y Vegetal de la UTEQ. C. fimbriata se reactivó mediante la metodología de sándwich de zanahoria, y luego de formados los peritecios,

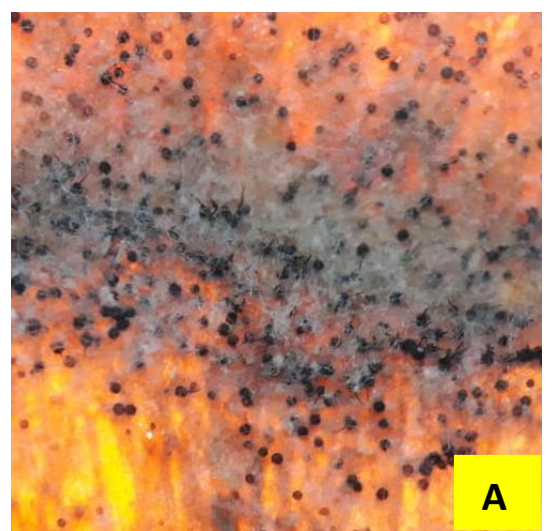

gotas de ascosporas acumuladas sobre las fimbrias que rodean el ostiolo fueron aisladas y sembradas en tubos de ensayo conteniendo medio de cultivo papa-dextrosa-agar (PDA) (Figura 1). Para ambos casos C. fimbriata y Fusarium spp. se repicaron en placas de Petri conteniendo $10 \mathrm{~mL}$ de medio de cultivo PDA + $0.2 \mathrm{~mL}$ de una mezcla de antibióticos $(50 \mu \mathrm{g} / \mathrm{mL}$ de penicilina y $25 \mu \mathrm{g} / \mathrm{mL}$ de estreptomicina $)^{5}$, e incubaron durante 192 horas (8 días) a $24 \pm 2{ }^{\circ} \mathrm{C}^{6}$. Mayores características taxonómicas de $C$. fimbriata aislada desde árboles enfermos de $T$. grandis se encuentran en Belezaca-Pinargote $e t$ al. $(2018)^{2}$.

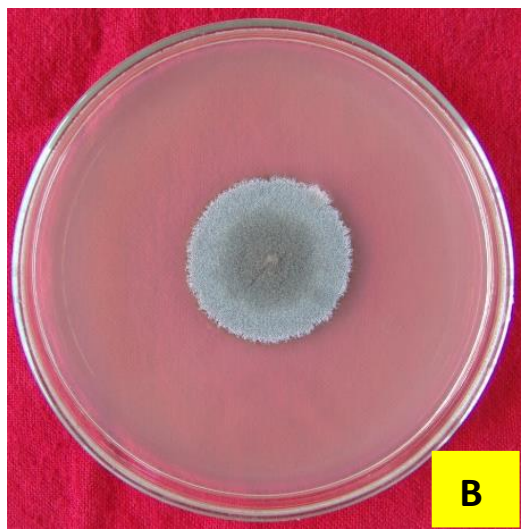

Figura 1. $\mathrm{A}=$ Peritecios de $C$. fimbriata formados durante el proceso de reactivación en sándwich de zanahoria. $\mathrm{B}=\mathrm{Colonia}$ pura de $C$. fimbriata en medio de cultivo PDA.

Las inoculaciones (pruebas de patogenicidad) se realizaron en el tallo de plantas de teca de cuatro meses de edad, con un diámetro a nivel del suelo de $1.5 \mathrm{~cm}$, en buen estado sanitario, procedentes del vivero de la Finca Experimental "La Represa", propiedad de la UTEQ. Para el efecto se desinfectó el área a cortar con algodón humedecido de alcohol, y empleando un bisturí estéril se realizó un corte inclinado que comprometió la corteza y xilema a $5 \mathrm{~cm}$ sobre el nivel del suelo. Dentro de la herida se aplicó un segmento de colonia del fitopatógeno seleccionado ( $0.5 \mathrm{~cm}$ de diámetro), previamente cortado en la placa de Petri con un sacabocado, y la herida conteniendo al hongo dentro, se cubrió con cinta de parafilm. Como control se inocularon plantas de teca bajo las mismas condiciones anteriores, con la diferencia que en lugar de inocular el patógeno, se aplicó dentro de la herida un segmento de agar-agar (inóculo, sin nutrientes).

Las plantas se regaron con agua limpia en función a las necesidades hídricas de las mismas. El experimento se estableció durante 45 días, tiempo en que se realizaron observaciones periódicas sobre el estado sanitario de las plantas, con el propósito de detectar la aparición de síntomas de la enfermedad de marchitez vascular, asociados al fitopatógeno inoculado, y registró detalladamente el desarrollo de la sintomatología en las plantas de teca. A los 45 días después de las inoculaciones las plantas fueron diseccionadas mediante cortes longitudinales y transversales para determinar y 
Ceratocystis fimbriata agente causal de la enfermedad de marchitez vascular de Tectona grandis L.f. (teca) en Ecuador Belezaca-Pinargote, $\mathrm{C}$.

medir daños o lesiones necróticas en tejidos, tanto en sentido ascendente y descendente, tomando como punto de referencia la parte central de la herida efectuada para la inoculación. Las áreas de necrosis fueron medidas en tres dimensiones (alto, ancho y profundidad) con el propósito de estimar el área aparente de necrosis, expresada en $\mathrm{cm}^{3}$.

\section{Tratamientos y Diseño Experimental.}

El experimento estuvo constituido por cuatro tratamientos: $\mathrm{T} 1$ = plantas de teca inoculadas con el fitopatógeno Ceratocystis fimbriata, $\mathrm{T} 2=$ plantas de teca inoculadas con el fitopatógeno Fusarium sp., T3 = plantas de teca inoculadas con la mezcla de los fitopatógenos Ceratocystis fimbriata + Fusarium sp. T4 = plantas de teca inoculadas únicamente con agar-agar (tratamiento control). Cada tratamiento estuvo conformado por 30 plantas. Se aplicó un Diseño Completamente al Azar (DCA).

\section{Análisis estadísticos.}

Los datos cuantitativos obtenidos se analizaron empleando herramientas de estadística descriptiva: media, desviación estándar, error estándar, coeficiente de variación, etc. Para establecer la existencia o no de diferencias estadísticas significativas entre tratamientos, los datos se analizaron bajo el esquema del análisis de varianza (ANOVA) con un nivel de significancia de 95\% $(P<0.05)$, previa comprobación de los supuestos de normalidad y homocedasticidad de varianzas. Posteriormente se aplicó la prueba LSD (mínima diferencia significativa), con un nivel de significancia del 95\% $(P<0.05)$. Para el efecto se empleó el paquete estadístico $S A S \quad 9.0$ versión para Windows.

\section{RESULTADOS}

\section{Volumen aparente de necrosis $\left(\mathrm{cm}^{3}\right)$.}

Se detectaron diferencias estadísticas significativas $(F=15.46 ; \quad P=0.000)$ entre los volúmenes aparentes de necrosis generados por los fitopatógenos inoculados (tratamientos) en las plantas de teca. Los tratamientos $C$. fimbriata, y C. fimbriata + Fusarium sp., generaron los mayores volúmenes aparentes de necrosis, con $1.52 \mathrm{~cm}^{3}$ y $1.93 \mathrm{~cm}^{3}$, respectivamente, siendo estadísticamente similares entre sí, pero diferentes a los tratamientos Fusarium sp., y el Control que alcanzaron volúmenes más bajos, de $0.27 \mathrm{~cm}^{3}$ y $0.16 \mathrm{~cm}^{3} \quad$ respectivamente, siendo estadísticamente iguales (Figura 2).

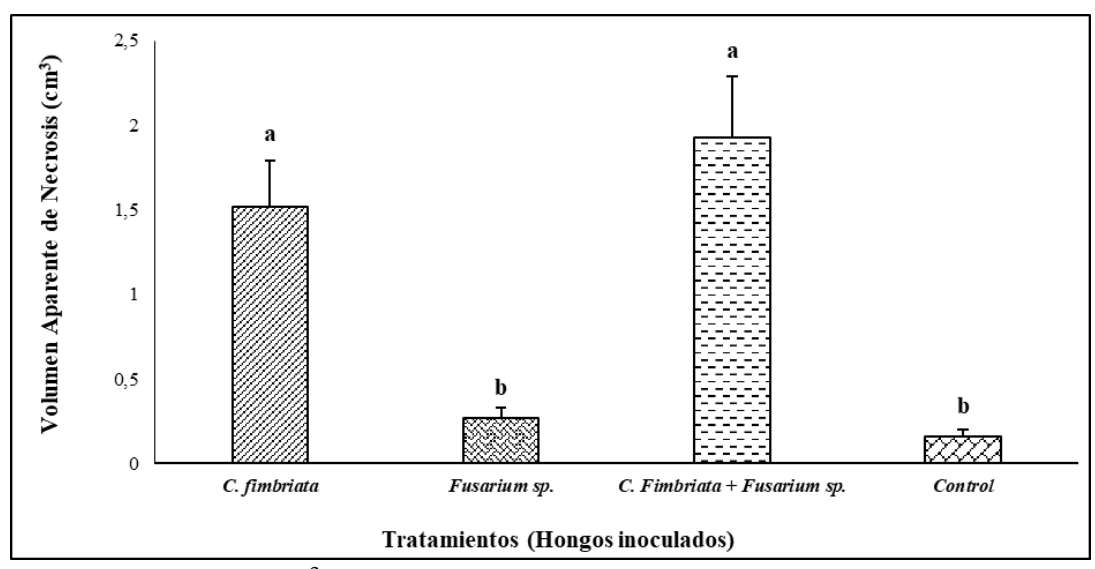

Figura 2. Volumen aparente de necrosis $\left(\mathrm{cm}^{3}\right)$ generado por hongos fitopatógenos (tratamientos) inoculados en plantas de $T$. grandis (teca) de 4 meses de edad, 45 días después de inoculadas a nivel de invernadero. Valores corresponden al volumen aparente de necrosis promedio de 30 plantas de teca, con su respectivo error estándar. 
Ceratocystis fimbriata agente causal de la enfermedad de marchitez vascular de Tectona grandis L.f. (teca) en Ecuador Belezaca-Pinargote, $\mathrm{C}$.

\section{Longitud total de necrosis $(\mathrm{cm})$.}

Se observaron diferencias estadísticas significativas $(F=15.99 ; \quad P=0.000)$ entre las longitudes de necrosis generadas por los hongos inoculados. Los tratamientos $C$. fimbriata, y $C$. fimbriata + Fusarium sp. produjeron las mayores longitudes de necrosis, con $5.41 \mathrm{~cm} \mathrm{y}$
$4.21 \mathrm{~cm}, \quad$ respectivamente, siendo estadísticamente similares entre sí, pero diferentes a los tratamientos Fusarium sp., y el Control que alcanzaron longitudes menores, de $1.75 \mathrm{~cm}$ y $1.11 \mathrm{~cm}$, respectivamente, y manteniendo un comportamiento estadístico similar (Figura 3).

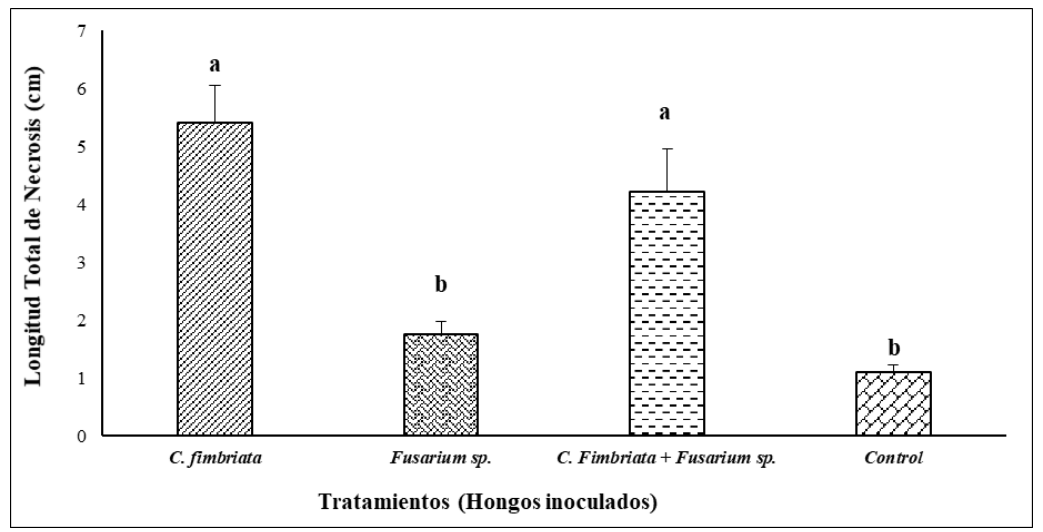

Figura 3. Longitud total de necrosis $(\mathrm{cm})$ generada por hongos fitopatógenos (tratamientos) inoculados en plantas de $T$. grandis (teca) de 4 meses de edad, 45 días después de inoculadas a nivel de invernadero. Valores corresponden a la longitud total de necrosis promedio de 30 plantas de teca, con su respectivo error estándar.

\section{Longitud ascendente $y$ descendente de necrosis.}

Para ambas variables se encontraron diferencias estadísticas significativas; longitud de necrosis ascendentes $(F=11.24 ; P=0.000)$ y descendente $(F=23.09 ; \quad P=0.000), \quad$ causadas por los fitopatógenos inoculados (tratamientos) en las plantas de teca. Los tratamientos C. fimbriata, y C. fimbriata + Fusarium sp., generaron las mayores longitudes ascendentes de necrosis, con $3.12 \mathrm{~cm} \mathrm{y} 2.59 \mathrm{~cm}$, respectivamente, y mantuvieron la misma tendencia, aunque presentaron valores menores para la longitud descendente, con $2.29 \mathrm{~cm}$ y $1.62 \mathrm{~cm}$ respectivamente, siendo en ambos casos estadísticamente similares entre sí, pero diferentes a los tratamientos Fusarium sp., y el Control que alcanzaron menores longitudes ascendentes de $0.73 \mathrm{~cm}$ y $0.48 \mathrm{~cm}$, y longitudes descendentes de $1.01 \mathrm{~cm}$ y $0.63 \mathrm{~cm}$ respectivamente, con un comportamiento estadístico similar entre ellos (Tabla 1)

Tabla 1. Longitud ascendente y descendente de necrosis $(\mathrm{cm})$ generada por la inoculación de hongos fitopatógenos (tratamientos) en plantas de $T$. grandis (teca) de 4 meses de edad, 45 días después de inoculadas a nivel de invernadero. Valores corresponden a longitudes ascendentes y descendentes de necrosis promedio de 30 plantas, con su respectivo error estándar.

\begin{tabular}{|c|c|c|c|c|}
\hline Tratamientos & $\begin{array}{c}\text { Longitud } \\
\text { ascendente }\end{array}$ & $\begin{array}{c}\text { Error } \\
\text { estándar }\end{array}$ & $\begin{array}{c}\text { Longitud } \\
\text { descendente }\end{array}$ & $\begin{array}{c}\text { Error } \\
\text { estándar }\end{array}$ \\
\hline Ceratocystis fimbriata & $3.12 \mathrm{a}$ & 0.56 & $2.29 \mathrm{a}$ & 0.16 \\
\hline Fusarium sp & $0.73 \mathrm{~b}$ & 0.14 & $1.01 \mathrm{c}$ & 0.12 \\
\hline Ceratocystis fimbriata + Fusarium sp. & $2.59 \mathrm{a}$ & 0.53 & $1.62 \mathrm{~b}$ & 0.22 \\
\hline Control & $0.48 \mathrm{~b}$ & 0.07 & $0.63 \mathrm{c}$ & 0.06 \\
\hline
\end{tabular}


Ceratocystis fimbriata agente causal de la enfermedad de marchitez vascular de Tectona grandis L.f. (teca) en Ecuador Belezaca-Pinargote, $\mathrm{C}$.

\section{Descripción de la sintomatología.}

Los síntomas presentados por las plantas de teca en los tratamientos $C$. fimbriata, y $C$. fimbriata + Fusarium sp. fueron similares, y para ambos casos entre los 11 y 18 días después de la inoculación se detectaron plantas con pérdida de coloración (clorosis) y turgencia en su sistema foliar. Fue notorio que, en las mismas plantas e igual periodo de tiempo, aparecieron brotes epicormicos a aproximadamente $2 \mathrm{~cm}$ bajo el punto de inoculación. A los 20 días posterior a la inoculación, plantas inoculadas con C. fimbriata, y C. fimbriata + Fusarium sp. con síntomas de enfermedad, murieron, quedando las hojas secas adheridas a la planta, y dos semanas después los brotes epicormicos también murieron (Figura 4).
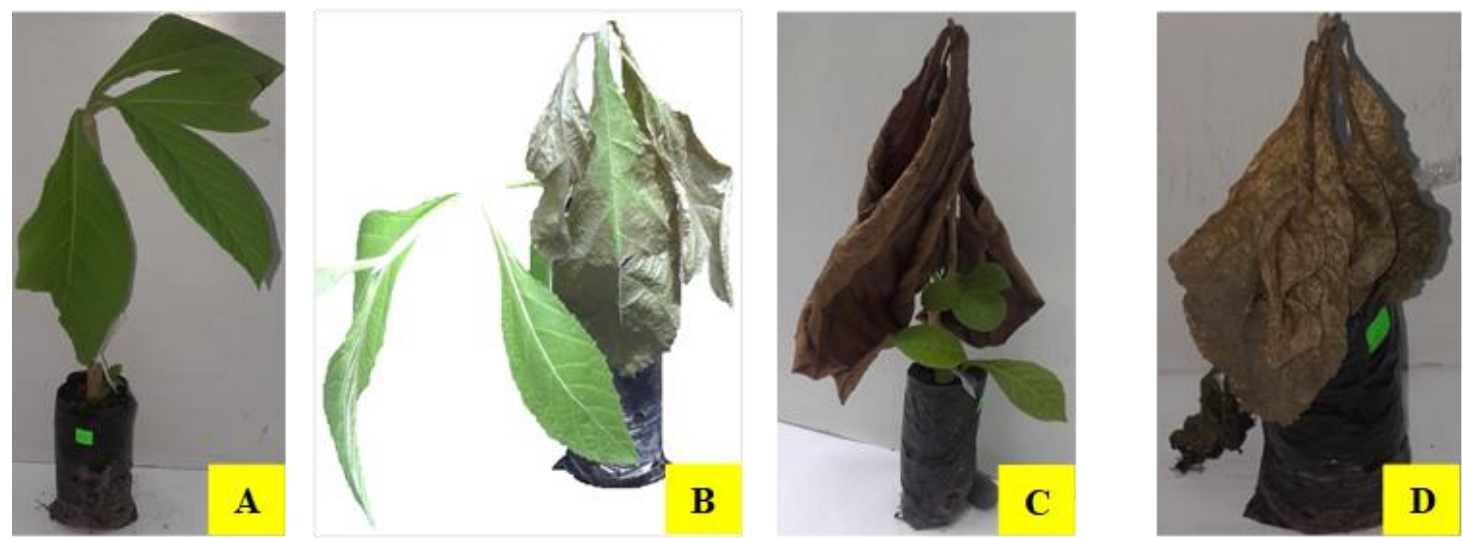

Figura 4. Sintomatología progresiva generada por la inoculación de microorganismos fungosos en plantas de teca a nivel de invernadero. Tratamientos: C. fimbrita, y C. fimbrita + Fusarium sp. A = Planta recién inoculada (sana), B $=$ Sistema foliar con presencia de marchitez vascular y generación de brotes epicormicos bajo el punto de inoculación, $\mathrm{C}=$ Sistema foliar muerto, cuyas hojas permanecen unidas a la planta, brotes epicormicos bajo el punto de inoculación, $\mathrm{D}=\mathrm{Planta}$ de teca con sistema foliar y brotes epicormicos muertos.

Las plantas inoculadas con Fusarium sp. al momento de la evaluación (45 días de incubación) no presentaron sintomatología visible de enfermedad. No se observó presencia de decoloración, marchitez, y pérdida de turgencia, etc., mostraron aspecto de plantas sanas, similares a las plantas control (Figura 5).
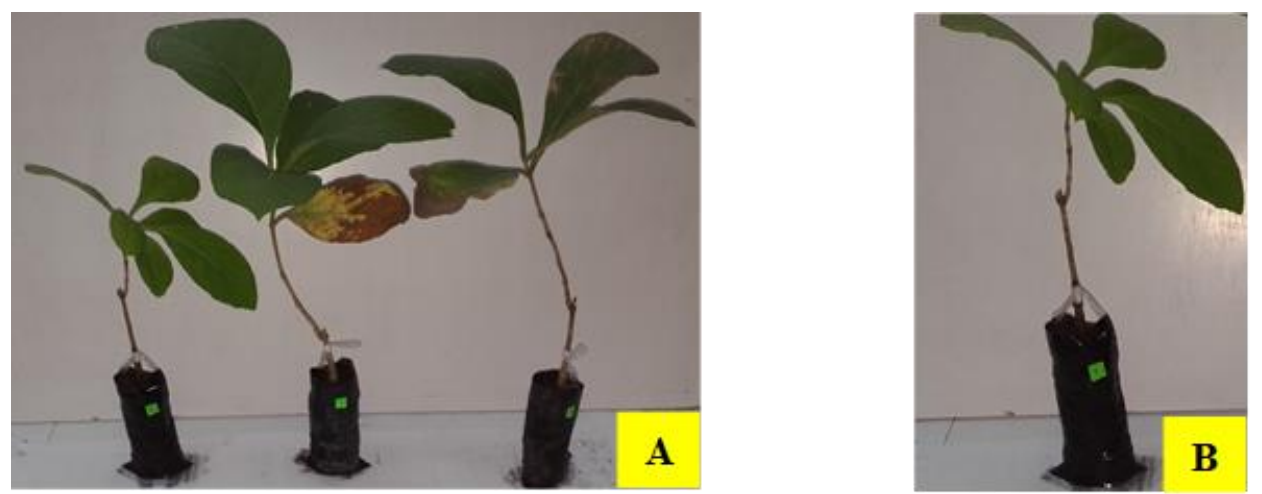

Figura 5. Plantas de teca inoculadas con (A) Fusarium sp. a los 45 días de incubación, sin presencia visible de síntomas de enfermedad, con aspecto similar a (B) las plantas control (sin inoculación de fitopatógenos). 
Ceratocystis fimbriata agente causal de la enfermedad de marchitez vascular de Tectona grandis L.f. (teca) en Ecuador Belezaca-Pinargote, $\mathrm{C}$.

\section{Recuperación de los microorganismos inoculados.}

Después de las evaluaciones, y dando cumplimiento a los postulados de Koch, a partir de tejidos necrosados se realizó la recuperación de los microorganismos inoculados en las plantas de teca. C. fimbriata fue recuperado mediante la técnica de "sándwich" de zanahoria, y Fusarium sp. en medio de cultivo PDA.

\section{DISCUSIÓN}

Hasta la década de 1990, las plantaciones de $T$. grandis existentes en el Trópico Húmedo Ecuatoriano (THE) no presentaban problemas sanitarios significativos, por lo que la especie era considerada altamente resistente a las duras condiciones ecológicas de la región. No obstante, a partir del año 2000 la masificación de plantaciones puras conllevó al aparecimiento de una compleja enfermedad que demandó un adecuado diagnóstico para así evitar se convierta en una problemática fitosanitaria recurrente y endémica ${ }^{4}$. Estudios a nivel de laboratorio daban cuenta de la constante presencia de Fusarium sp. y $C$. fimbriata en árboles enfermos ${ }^{2,7}$.

Postulados de Koch permitieron determinar que los volúmenes aparentes de necrosis generados por Fusarium sp. en plantas inoculadas fueron similares a los producidos por el control (agar agar) lo cual indicaría que este hongo o cepa del microorganismo no es patogénico para $T$. grandis. Sin embargo, aquellas plantas inoculadas con C. fimbriata, y $C$. fimbriata + Fusarium sp. generaron volúmenes aparentes de necrosis muy significativos que incluso derivaron en la muerte de varias plantas inoculadas. Similar comportamiento mostraron los tratamientos con relación a la longitud total de necrosis. Es de destacar que las necrosis en plantas inoculadas con $C$. fimbriata, y $C$. fimbriata + Fusarium sp., fueron mayores en sentido ascendente, a diferencia del tratamiento Fusarium sp. cuyas necrosis en plantas inoculadas fueron mayores en sentido descendente, lo cual indicaría que $C$. fimbriata prefiere colonizar y necrosar tejidos vasculares desde el punto de infección (ingreso) hacia arriba, situación que ya fue detectada y reportada en árboles de teca de plantaciones comerciales a nivel de campo ${ }^{2,4,7}$.

Estos resultados serían un indicador de la alta patogenicidad que posee $C$. fimbrata en $T$. grandis, lo cual no es de sorprender, ya que este ascomicete tiene un amplio rango de hospedadores y se lo ha encontrado causando enfermedades en especies leñosas en Ecuador y otras regiones del planeta ${ }^{8}$. C. fimbriata ocasiona la enfermedad de "mal de machete" en Theobroma cacao en Latinoamérica", "marchitez vascular y pudrición del fuste" en Schizolobium parahybum ${ }^{\mathbf{1 0}}$,"marchitez vascular y pudrición del fuste" en Acrocarpus fraxinifolius ${ }^{\mathbf{1 1}}$, "pudrición del fuste y marchitez vascular" en Gmelina arbórea ${ }^{\mathbf{1 2}}$, para el caso de Ecuador, "marchitamiento vascular" del Eucaliptus spp. en Brasil ${ }^{13}$, etc. Pese que en Ecuador varios investigadores habían encontrado a $C$. fimbriata asociado a árboles enfermos de $T$. grandis, su implicación en la patogénesis de la enfermedad no había sido dilucidada científicamente ${ }^{2,4,7,14,15}$, hasta ahora.

\section{CONCLUSIONES}

De acuerdo a los resultados obtenidos el agente causal de la enfermedad de "marchitez vascular" en $T$. grandis sería Ceratocystis fimbriata Ellis $\&$ Halst. Este es el primer reporte de $C$. fimbriata causando patogénesis en plantaciones comerciales de teca en Ecuador.

\section{AGRADECIMIENTOS}

Al proyecto de investigación PFOC 5-21-2017 "Determinación de problemas fitosanitarios presentes en plantaciones de teca (Tectona grandis) de las provincias Santo Domingo de Los Tsáchilas, Los Ríos, Guayas y Esmeradas. 
Ceratocystis fimbriata agente causal de la enfermedad de marchitez vascular de Tectona grandis L.f. (teca) en Ecuador Belezaca-Pinargote, $\mathrm{C}$.

\section{REFERENCIAS}

1. Kaosa-ard A. Teak (Tectona grandis L.f.) its natural distribution and related factors. Nat. Hist. Bull. Siam. Soc. 1989; 29:55-74.

http://www.siameseheritage.org/nhbsspdf/vol021030/NHBSS_029_g_KaosaArd_TeakTectonaG rand.pdf

2. Belezaca-Pinargote C, Solano-Apuntes E, López-Tobar C, Baque-Mite R, Ávila-Loor A, Cóndor-Jiménez M, Bohórquez-Barros T, Dueñas-Alvarado D. Hongos fitopatógenos asociados a la enfermedad de marchitez vascular y muerte regresiva en plantaciones de Tectona grandis L.f. (teca) en el Trópico Húmedo Ecuatoriano. Boletín Micológico. 2018; 33(2):17-29.

https://micologia.uv.cl/index.php/Bolmicol/arti cle/view/1410

3. Kollert W, Cherubini L. Teak resources and marked assessment 2010 (Tectona grandis Linn. F.). Food and Agriculture Organization of the United Nations. Forestry Department. Planted Forest and Trees Working Papers Series FP/47/E FAO, Rome, Italy. 2012; 42 p. http://www.fao.org/3/a-an537e.pdf

4. Ávila-Loor, AA. Identificación de microorganismos fungosos asociados a la enfermedad de muerte regresiva en plantaciones de Tectona grandis L.F. (teca) en la zona central del Trópico Húmedo Ecuatoriano. Proyecto de Investigación de Ingeniero Forestal. Universidad Técnica Estatal de Quevedo, Ecuador. 2016; 63 p.

5. Parkinson D. Filamentous fungi. In Weaver R, Angle S, Bottomley P, Bezdicek D, Smith S, Tabatabai A, Wollum A. (eds). Methods of Soil Analysis. Part 2, Microbiological and Biochemical Propeties. Number 5 in Soil Science Society of America Book Series. Soil
Science Society of America. Inc., Madison, Wisconsing, USA. 1994; $329-350 \mathrm{p}$.

6. CMI (Commonwealth Mycological Institute). Plant pathologist's Pocketbook. London. England. 1983; 267 p.

7. Solano-Apuntes EH, Belezaca-Pinargote CE, López-Tobar RM, Macías-Suárez KP. Incidence and severity of vascular wilt disease, regressive death of Tectona grandis L. F. in four provinces of Ecuador. Universidad y Sociedad. 2019; 11(5):262-269.

https://rus.ucf.edu.cu/index.php/rus/article/view $\underline{/ 1371 / 1399}$

8. Ferreira MA, Harringtong TC, Alfenas AC, Mizubuti ES. Movement of genotypes of Ceratocystis fimbriata within and among Eucalyptus plantations in Brazil. Phytopathology. 2011; 101(8):1005-1012. https://www.ncbi.nlm.nih.gov/pubmed/2148614 $\underline{4}$.

9. Harrington TC. Ceratocystis diseases. In Infectious Forest Diseases. Eds. Gonthier P, Nicolotti G. CABI. 2013; 230-255 p. ISBN: 139781-78064-0402.

10. Belezaca C, Suárez C, Vera D. Hongos fitopatógenos asociados a la enfermedad de muerte regresiva y pudrición del fuste de pachaco (Schizolobium parahybum) en el Trópico Húmedo Ecuatoriano. Boletín Micológico. 2011; 26 (1): 15-22. https://revistas.uv.cl/index.php/Bolmicol/article /view/895/872

\section{Belezaca $C$, Mora $W$, Prieto $O$, Cedeño $P$,} Moran J, Valenzuela E. Hongos asociados a problemas fitosanitarios emergente en especies forestales de importancia económica del Trópico Húmedo Ecuatoriano. In Libro de resúmenes del XXI Congreso de la Sociedad Chilena de Fitopatología. Puerto Varas, Chile $(17-19$ de octubre del 2012). 2012; p 79. 
Ceratocystis fimbriata agente causal de la enfermedad de marchitez vascular de Tectona grandis L.f. (teca) en Ecuador Belezaca-Pinargote, $\mathrm{C}$.

https://www.sochifit.cl/resumen/xxi-congresode-fitopatologia-octubre-2012/\#Articulo_43

12. Macías-Moncayo ML. Determinación del agente causal de la enfermedad de marchitez vascular y pudrición del fuste de Gmelina arborea Roxb. en el Trópico Húmedo Ecuatoriano. Proyecto de Investigación de Ingeniera Forestal. Facultad de Ciencias Ambientales. Universidad Técnica Estatal de Quevedo, Ecuador. 2019; 47p.

http://biblioteca.uteq.edu.ec/cgi-bin/koha/opacdetail.pl?biblionumber $=16890$

13. Ferreira MA, Alfenas AC, Mafia RG. Ceratocystis fimbriata em espécies florestais e agronômicas no Brasil. Pages 75-101 in: Patologia florestal: Desafios e perspectivas. NEFIT. 1 ed. Lavras, MG. 2013. https://www.researchgate.net/profile/Maria_Fer reira42/publication/324784433_Ceratocystis_fi mbriata_em_especies_florestais_e agronomica s_no_Brasil/links/5b19a256a6fdcca67b65f816/ Ceratocystis-fimbriata-em-especies-florestais-eagronomicas-no-Brasil.pdf

14. Cóndor-Jiménez, MF. Determinación del agente causal de la enfermedad de marchitez vascular y muerte regresiva de Tectona grandis L.f. en el Trópico Húmedo Ecuatoriano. Proyecto de Investigación de Ingeniera Forestal. Facultad de Ciencias Ambientales. Universidad Técnica Estatal de Quevedo, Ecuador. 2017; $55 \mathrm{p}$.

http://repositorio.uteq.edu.ec/handle/43000/201 $\underline{5}$

15. Flores T, Crespo R, Cabezas F. Plagas y enfermedades en plantaciones de Teca (Tectona grandis L. f.) en la zona de Balzar, provincia del Guayas. Ciencia y Tecnología. 2010; 3(1): 1522.

http://revistas.uteq.edu.ec/index.php/cyt/article/ view/41 